\title{
LA-UR-18-20836
}

Approved for public release; distribution is unlimited.

Title: Introduction to identiFINDERs

Author(s): $\quad$ Rees, Brian G.

Intended for: Training presentation

Issued: 
Disclaimer:

Los Alamos National Laboratory, an affirmative action/equal opportunity employer, is operated by the Los Alamos National Security, LLC for the National Nuclear Security Administration of the U.S. Department of Energy under contract DE-AC52-06NA25396. By approving this article, the publisher recognizes that the U.S. Government retains nonexclusive, royalty-free license to publish or reproduce the published form of this contribution, or to allow others to do so, for U.S. Government purposes. Los Alamos National Laboratory requests that the publisher identify this article as work performed under the auspices of the U.S. Department of Energy. Los Alamos National Laboratory strongly supports academic freedom and a researcher's right to publish; as an institution, however, the Laboratory does not endorse the viewpoint of a publication or guarantee its technical correctness. 


\section{Introduction to IdentiFINDERS}

Brian Rees

Los Alamos National Laboratory 

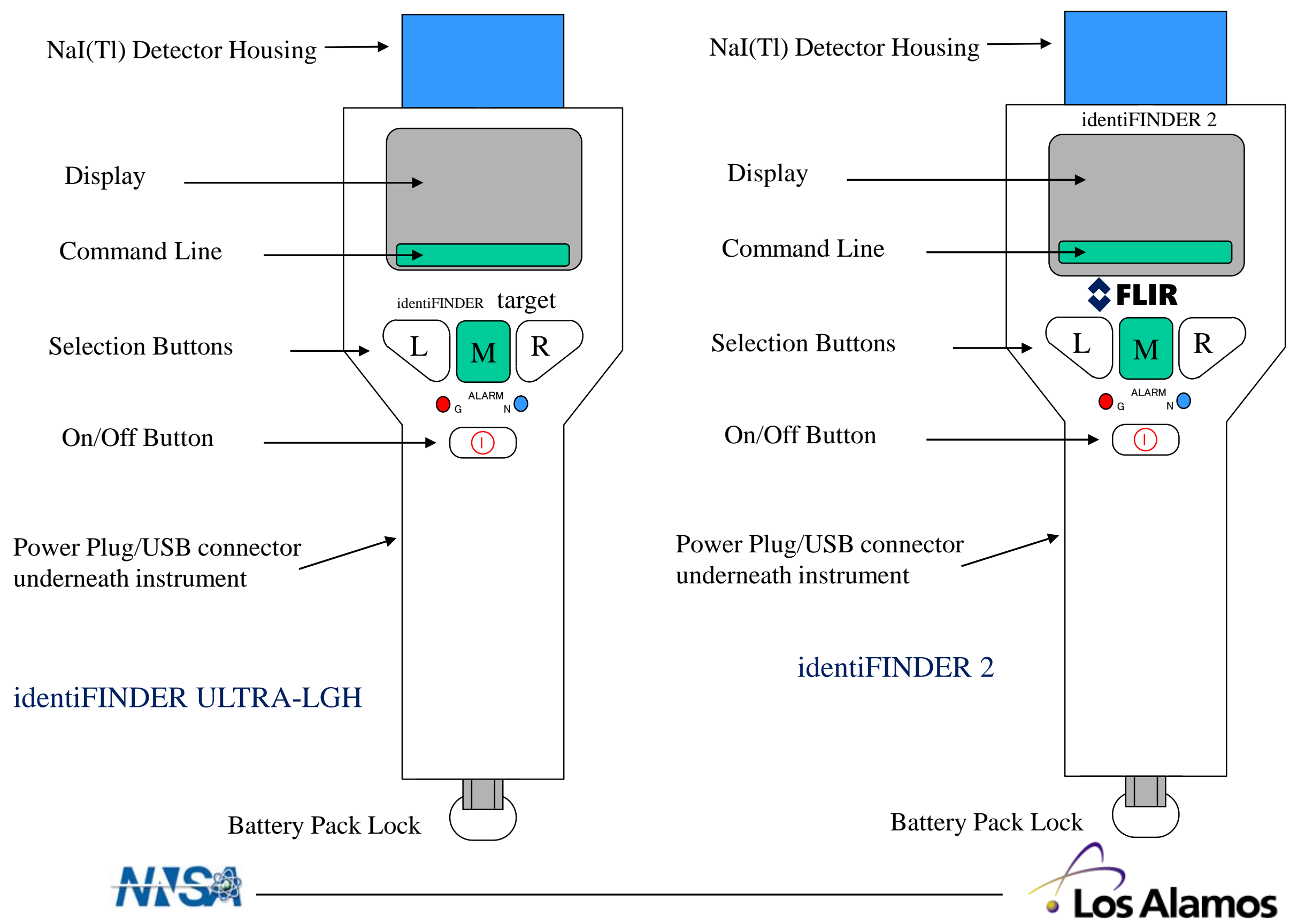


\section{Overview}

- The identiFINDER has three detectors; a 1" by 2" $\mathrm{NaI}$ crystal for spectroscopy, a He3 tube for neutron detection, and a GM tube for higher dose rates $(1-40 \mathrm{mr} / \mathrm{hr})$

- It provides information on radiation levels

- It can automatically identify isotopes in its library

- It can save spectra for transfer to a computer

- 4-8 hr battery life 


\section{Operating Modes}

- FINDER

- IDENT or Identify - Identification

- Dose Rate

- Command line shows the other two modes and the OPTIONS button

- Advanced mode - spectrum 


\section{Overview}

- Before turning the unit on

- Check it for visible damage

- Ensure rechargeable batteries are installed before connecting the battery charger

- Standard batteries can also be used, but connecting the charger when non-rechargeable batteries are installed can lead to explosion

- Turn on the unit in low background areas if at all possible

- Different tones for gamma, neutron, alarms

- Can vibrate at warning or alarm levels 


\section{Getting Started}

- Calibration should take about 90 - 200 seconds

- Longer calibration times or erratic line movement may mean that the background levels are too high

- Proper calibration enables the system to identify the energy of other peaks in the spectrum and therefore other radioactive materials in the environment

- The system knows what count rate it should see from the internal calibration source 


\section{Getting Started}

- When calibration is complete, the display will read "Calibration successful

- The system will automatically go into dose rate mode when calibration is complete

- If the user presses EXIT during the calibration, the previous calibration settings will be used

- This should only be done when necessary

- A sufficiently clean background area is not available

- The system needs to be put into use as quickly as possible 


\section{Finder Mode}

- Finder mode is useful for finding sources

- Measures bkgd for 10 seconds before starting

- The most recent reading will be indicated at the bottom of the running histogram

- All readings and plots are background subtracted

- The histogram mode will continue until "EXIT" is pressed

- User can change directly to Identification Mode, or Spectrum Mode (through “options”)

- Height will autoscale 


\section{Search}

- One of your best search tool is your eyes

- "IJDLR"

- Use "Tees"

- Proceed along one direction, rate increases, then decreases

- Return to highest point

- Search at $90^{\circ}$

- Iterate until it's found

- Remember Distance and Shielding

- Ensure no other sources are present 


\section{Identification Mode}

- Press “Identify"

- Identification is crucial to proper response

- It needs to be accurate and happen as soon as practicable

- Display shows

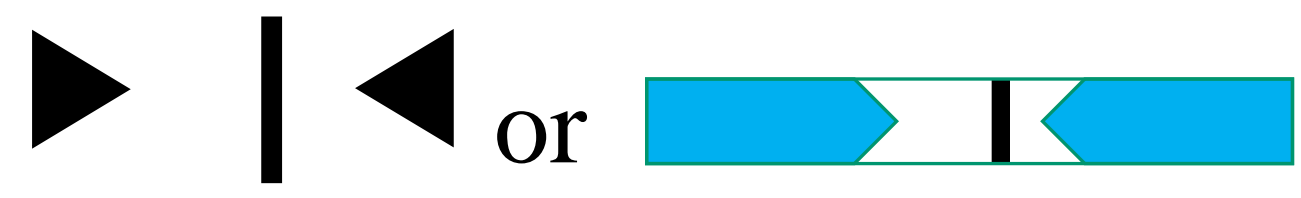

- Keep center bar between arrows, or as high a count rate as practicable

- Follow instructions given 


\section{Identification Mode}

- If the system does not have enough data to make a good identification, it will say "Low Activity! Do you want to continue?”

- "Yes" instructs the system to take additional data, which may give it enough information to make a good identification

- "No" cancels the identification and returns the system to the starting point of the identification 


\section{Identification Mode}

- After the spectrum collection time is over, the system analyzes the spectrum

- The result is displayed as a confidence value and an isotope

$$
8 \quad \text { Cs- } 137
$$

- The confidence value is a number between 1 and 10 (11 for Spinal Top mode); the higher the number, the more certain the system is of the identification

- The IdentiFINDER2 can also provide class (Medical, etc.) and Severity (Innocent, etc.) if selected 


\section{Identification Mode}

- The more isotopes listed, and the more low numbers you see, the more you need to download the spectrum and have spectrum analyzed by an expert, something may be getting lost in the mix

- "Nuclide Listing: Not in Library"

- Remember that the system can only identify isotopes that are in the list it's using; if there is something around that is not on a list, you'll never know without examining the spectrum by hand 


\section{Dose \& Rate \\ Warning \& Alarm}

- Think of the dose alarm and warning as an odometer

- Think of the rate alarm and warning as a speedometer

- Think of the warning as a message that you are getting close to an alarm

- Think of an alarm as a message that you have exceeded a limit (depending on where the alarm was set)

- Analogy: driving a car when you've been ordered to stay within $\mathrm{x}$ miles from home station 


\section{Options (Ultra LGH)}

- Gamma/N counting

- Reset accumulated gamma dose

- Stop counts, reset counts

- Finder options

- Response time

- Sensitivity

- Doserate options

- Integration time

- Units

- Alarm options

- Alarm Indications

- Sound on/off

- Vibrate on/off

- Light on/off

- $\quad$ Dose Alarm level

- Alarm

$$
\text { - Warning }
$$

- $\quad$ Dose Rate Alarm

$$
\text { - } \quad \text { Alarm }
$$

- Neutron Alarm level

- Neutron count rate alarm
- More options

- Show Spectrum

- Identify Options

- Identify Settings

$$
\begin{aligned}
& \text { Time (s) } \\
& \text { Number of Nuclides } \\
& \text { Library } \\
& \Rightarrow \quad \text { Security } \\
& " \quad \text { Customs } \\
& " \quad \text { Industrial } \\
& " \quad \text { Medical } \\
& " \quad \text { Nuclear } \\
& " \quad \text { OSI }
\end{aligned}
$$

Edit Isotope list

- Time Presets

- Real

- Live

- Do Calibration

- Advanced Options

- Setup Display/Time

- Maintenance

Change Password

Show Status

Factory Defaults

Erase all Spectra Factory tests

- Stabilization

- Calibration Source

- Data Logging 


\section{Options (Identifinder2)}

\section{Advanced Menu}

- More options

- Spectrum (shows it)

- Identification Options

- Identification Settings

- Nuclide Visibility

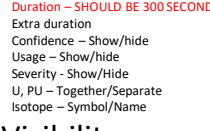

- Nuclide Usage (IND - Industrial, etc)

- Nuclide Severity - Innocent/Suspicious/Threatening

- Presets

- Real Time - No Limit

- $\quad$ Live time - No Limit

- Counts - No Limit

- Recalibrate

- $\quad$ Bluetooth SPP

- $\quad$ Advanced Options

- General Options

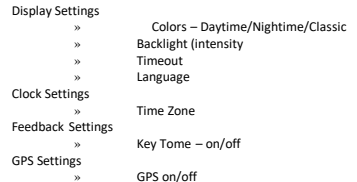

- Maintenance

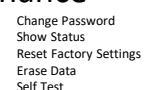

- Data Logging

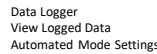

- Connectivity options

$$
\begin{aligned}
& \text { Send Data } \\
& \text { Send Identification } \\
& \text { S" Send Spectrum } \\
& \text { Bluetooth Periphery } \\
& \text { Remote Server Setting } \\
& \text { Mass Strorage } \\
& \text { Network } \\
& \text { Reports } \\
& \text { Downlload settings (.n42, spc, etc.) }
\end{aligned}
$$

- Gamma, Neutron Counters

- Accumulated Dose, gamma and neutron counts

- $\quad$ Finder Settings

- Available?

- $\quad$ Time (e.g. 1s/10)

- Sensitivity (e.g. 100\%)

- $\quad$ Dose Rate Settings

- Integration Time

- Displayed Unit

- Neutron (cps/cpm)

- Alarm Options

- Alarm Indicators

- LEDs

- Beeper

- Vibrator

- Pop-up Screen

- Dose Alarm Thresholds

- Alarm

- Warning

- $\quad$ Dose Rate Alarm Thresholds

- Alarm

- Warning

- Neutron Alarm Thresholds

- Alarm (\# of counts)

- Integration time

- Warning (\# of counts)

- Integration time

- Current Alarm State 


\section{Options (identiFINDER2) cont}

- Recalibrate

- Clock Settings

- Time Zone

- Current Alarm State

- Accumulated dose

- Current dose rate

- Current Neutron count rate

- Connectivity Options

- Same options as under Advanced Menu/More options/Advanced Options

- When in Spectrum mode:

- Mark Channel - Mark a channel

- $\quad$ Clear Markers

- 4Cursor

- Cursor $>$

- Save

- $\quad$ Load - Load a different spectrum to screen

- $\quad$ Identify - Shows nuclides ID'd

- $\quad$ Corrected/Raw Display

- $\quad$ Start/Stop - allows for > preset time

- $\quad$ Zoom 3 MeV/Zoom 1.5 MeV/Zoom 1:1/Zoom ROI

- $\quad$ Clear - Clears current spectrum

- $\quad$ Ymax/4/Ymax/2 / Linear/ Square Root/Logarithmic

- Channel/Energy

- Calibration

- $\quad$ Live time/Real Time 


\section{General Procedure}

- Use to finder mode and locate the source(s)

- If the dose rate is very high, go straight to identification mode

- Use identification mode to identify sources

- Use Advanced Mode - Spectrum to see the analysis results 